\title{
The Bio-Oscillator: A Circuit for Cell-Culture Assays
}

\author{
G. Huertas, A. Maldonado, A. Yúfera, A. Rueda, and J. L. Huertas
}

\begin{abstract}
A system for cell-culture real-time monitoring using an Oscillation-Based (OB) approach is proposed. The system transforms a Cell-Culture Under Test (CCUT) into a suitable "biological" oscillator, without needing complex circuitry for excitation and measurement. The obtained oscillation parameters are directly related to biological test, owed to an empirically extracted cell-electrode electrical model. A discrete prototype is proposed and experimental results with living cell culture are presented, achieving the expected performances.
\end{abstract}

Index Terms- Bioimpedance sensors, microelectrode, Electrical Cell-substrate Impedance Spectroscopy (ECIS)

\section{INTRODUCTION}

$\mathrm{M}$ ANY BIOLOGICAL parameters and processes can be sensed and monitored using their electrical impedance as a marker [1], with the advantage of being a noninvasive and relatively cheap technique. Cell growth, cell activity, or cell motility, are processes which can be detected with microelectrode-cell impedance sensors [1-5]. Among Impedance Spectroscopy (IS) techniques, Electrical Cellsubstrate Impedance Spectroscopy (ECIS) was proposed in 1986 [5] as an alternative method for experimentation in cell culture assays. Several works have taken advantage for this impedance-based cell culture real-time monitoring technique [4-6], allowing to greatly decreasing both the cost and the human effort in such assays, and reducing the result dispersion since can employ only one culture by assay.

Classical ECIS measurements require a two-electrode setup (sensing and GND) and an AC current source $\left(i_{x}\right)$ for excitation at several frequencies. By measuring the voltage response $\left(v_{x}\right)$, the impedance $\left(Z_{x}\right.$ : magnitude and phase) of the two-electrode plus cell-culture system at a given frequency is calculated. Fig. 1 shows an example of ECIS electrode from [12]. Impedance changes are originated from cell adhesion to the bottom surface, and its value increasing with the number of cells attached to such a bottom. The measured impedance is dependent on the electrode technology and geometry, the electrical cell-electrode model [4-6] and the working frequency.

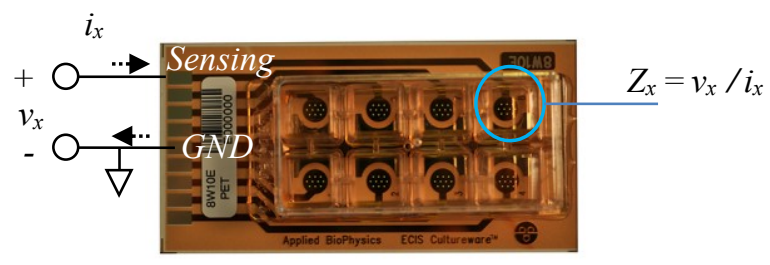

Fig. 1: The 8E10W system. Each system has 8 separated wells for cell culture and 10 electrodes of 250 um diameter by well [12].
The concept here proposed aims to reduce the required circuitry for impedance measurements, avoiding the need of any input stimuli. As a consequence, the test setup procedure is improved and simplified by applying the so-called Oscillation-Based (OB) method [8]. This approach converts the Cell Culture Under Test (CCUT) into an oscillator, adding just a few extra external components to force the "biological circuit" to oscillate. Any variation inside the CCUT (in time) will generate changes in the frequency/amplitude and consequently, these alterations will become observable. This technique avoids the need of complex resources for stimuli generation as well as means a reduction and simplification in the impedance measurement circuits [2-4].

The rest of the paper is organized as follows. Section II gives a brief introduction on the electrical electrode sensor model and its relevance to ECIS experiments. Section III presents the proposed bio-oscillator circuit able to extract the value of the cell-culture area from the electrical oscillation parameters (frequency and amplitude). Section IV describes the discrete electronic system employed for test. Finally, in Section V, the experimental results obtained using several test loads are shown.

\section{THE CELL-ELECTRODE SENSOR}

The impedance under test of a two-electrode system like every one of those shown in Fig $1[6,7]$ has several elements. For an electrode in saline solution, we have to consider the double layer capacitance $\left(C_{d l}\right)$, the transfer resistance $\left(R_{p}\right)$ and the spreading resistance $\left(R_{S}\right)$ for electrodes non-covered by cells. When any cell attachment happens, a new circuit element, the gap resistance $\left(R_{\text {gap }}\right)$, models this effect [7]. The model is valid only for AC voltages with amplitude below 50$100 \mathrm{mV}$ [3]. In this work, it has been considered the use of circular gold microelectrodes of $250 \mu \mathrm{m}$ diameter, which can be totally or partially covered by cells in the culture. Henceforth, the fill factor $(f f)$ parameter represents the per cent of the electrode area $(A)$ covered by cells $\left(A_{c}\right)$.

The sensor impedance for a given electrode covered by cells, $Z_{\text {cell-electrode }}(s)$, can be analyzed from the experimental set-up shown in Fig. 2, where we have included the circuit model employed for the electrodes. The $R_{\text {in }}$ resistor maintains the current flowing across the cell-electrode in adequate signal levels (below $20 \mu \mathrm{A}$ for cell protection, and $50-100 \mathrm{mV}$ for electrode modeling constrains [3]). The Bode plot for this bioelectronic system $H_{z}(s)$ (magnitude and phase) is shown in Fig. 3, where curves for several cell occupation areas, $f f$, have been represented. Let us notice that when consider frequencies around $10^{4} \mathrm{rad} / \mathrm{s}$, the system has optimum $\mathrm{ff}$ sensitivity. This means that both, magnitude and phase response, can be correlated to the fill factor parameter or cell-to-electrode 
overlap area, $A_{c}$. Most ECIS techniques search for the best frequency response for optimum impedance characterization, and then, perform the measurements knowing the $f f$ dependence. Usually, absolute magnitudes (Fig.3) or normalized magnitudes are employed as sensitivity curves for this kind of impedance sensors. Instead, in our case, we will use the magnitude and phase curves of the bio-electrical model for $H_{z}(s)=-Z_{\text {cell-electrode }}(s) / R_{i n}$, in Fig. 2 and 3, to force an oscillatory behavior which can be straightforward correlated to the cell-to-electrode area overlap.

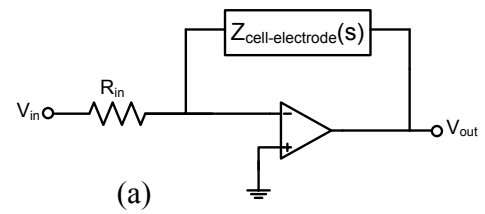

$\mathbf{Z}(\omega) / \mathbf{A} \quad \mathbf{R}_{\mathrm{s}}$

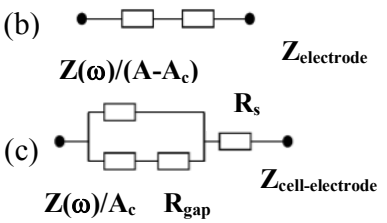

Fig. 2: (a) Circuit proposed for cell-electrode impedance testing. (b) Circuit model for a bare electrode of area A. (c) Circuit model for an electrode of area A, partially covered with cells, an area $A_{c}$. Estimated values for a $50 \times 50 \mu \mathrm{m}^{2}$ gold electrode: $\mathrm{R}_{\mathrm{s}}=5.4 \mathrm{k} \Omega, \mathrm{Z}(\omega)=\mathrm{C}_{\mathrm{dl}}|| \mathrm{R}_{\mathrm{p}}$, with $\mathrm{C}_{\mathrm{dl}}=0.37 \mathrm{nF}$ and $\mathrm{R}_{\mathrm{p}}=25 \mathrm{M} \Omega$. $\mathrm{R}_{\text {gap }}=75 \mathrm{k} \Omega$.

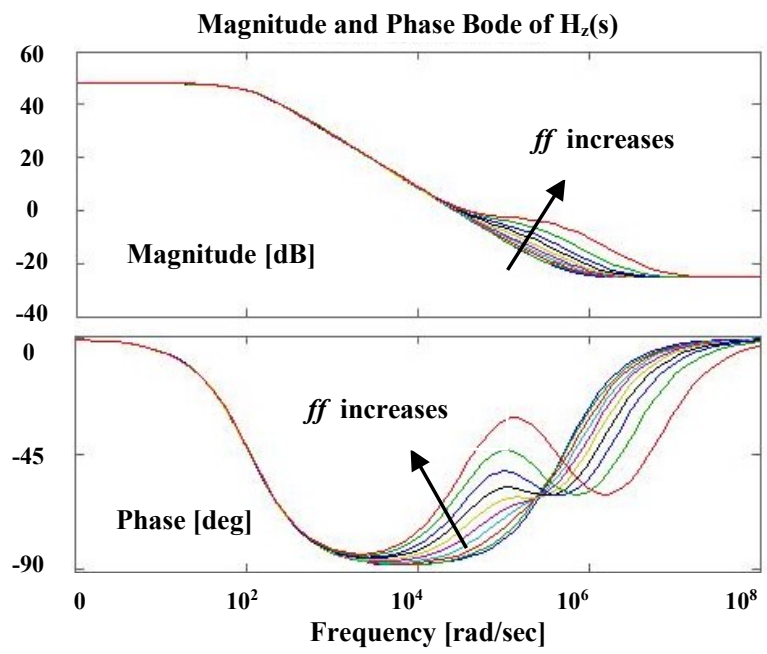

Fig. 3: Bioelectronics system Bode curves for $H_{z}(s)=-Z_{\text {cell-electrode }} / R_{\text {in }}$ using several cell-culture occupation areas $(f f)$.

As a sensor sensitivity test, cell culture experiments were done with the $8 \mathrm{~W} 10 \mathrm{E}$ system and the cell line U2OS, which was isolated from a mild osteosarcoma in 1964. At chromosomal level, its karyotype it is greatly altered, with a ploidy in the range of hypertriploid. In culture, these cells grow as an adherent monolayer. When seeded at low density, these cells have a tendency to grow forming discrete colonies. The objective of these assays was to evaluate the sensitivity of the ECIS sensor. Eight wells were seeded with a) only medium (2 wells), b) 500 cells (2), c) 1000 cells (2) and d) 2000 cells (2). Fig. 4 shows the image of the cell cultures after a 5-days growth. The transfer function $H_{z}(s)$ was measured (magnitude and phase) by using a network analyzer. The phase response is shown in Fig. 5, illustrating how at $8 \mathrm{kHz}$, the number of cells (fill-factor) can be estimated by measuring that parameter (the phase response) at $V_{\text {out }}$ (see Fig. 2). Table I summarizes the experimental data at $8 \mathrm{kHz}$, achieving a sensor sensitivity as expected; it is, magnitude and phase correlates with the cell population.
Table I: Magnitude and phase response at $8 \mathrm{kHz}$ ( 5 days growing).

\begin{tabular}{|l|c|c|}
\hline Cells at time $=0$ & Magnitude & Phase $(\mathrm{deg})$ \\
\hline Medium $($ no cells $)$ & $-68 \mathrm{~dB}$ & 90 \\
\hline Low $(500$ cells $)$ & $-67 \mathrm{~dB}$ & 93 \\
\hline Int $(1000$ cells $)$ & $-66 \mathrm{~dB}$ & 108 \\
\hline High $(2000$ cells $)$ & $-61 \mathrm{~dB}$ & 140 \\
\hline
\end{tabular}
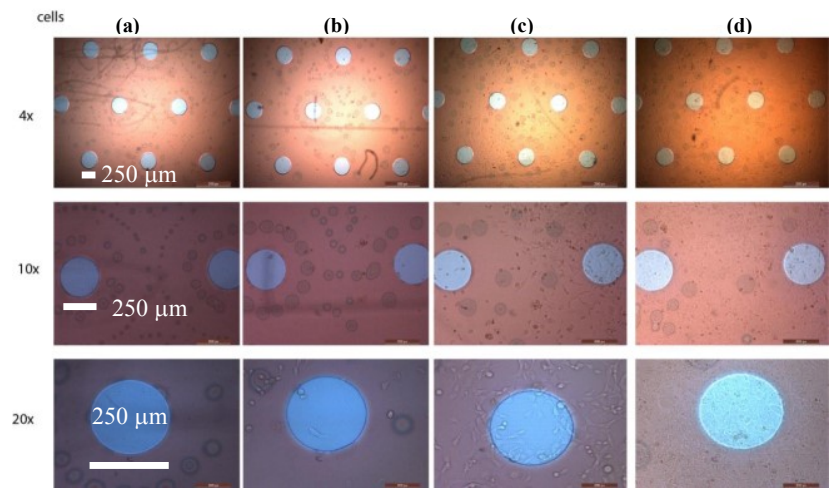

Fig. 4: Cell culture images showing four well with (a) only medium, (b) 500 initial cells, (c) 1000 initial cells and (d) 2000 initial cells, at different zooms.

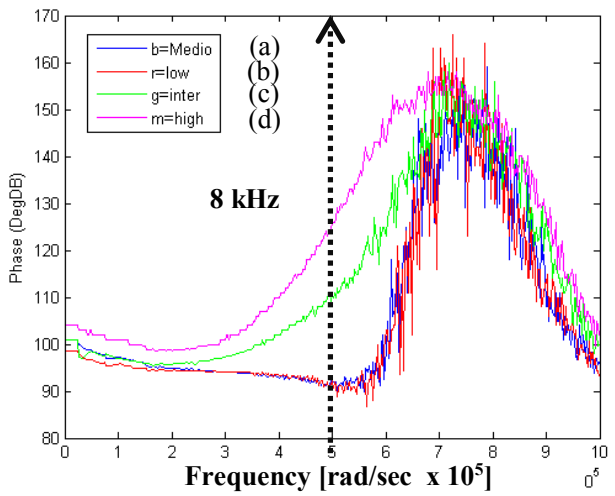

Fig. 5: Experimental phase response obtained for (a) medium, (b) 500 cells assay,(c) 1000 cells assays and (d) 2000 cells assays, after 5 days growing.

\section{Proposed Bio-Oscillator Circuit BASED System}

The key idea of the proposed measurement system relies on transforming the CCUT in a robust oscillator, by adding some extra components. Fig. 6 shows the block components to perform the proposed transformation. In order to force oscillations, a positive feedback loop has to be added. From the point of view of the OB application, it is particularly important an accurate prediction of the oscillation parameters (frequency and amplitude), either analytically or by means of simulations [9-11]. It is also necessary to avoid the dependency of these parameters upon the saturation characteristics of the active elements, like it occurs in many

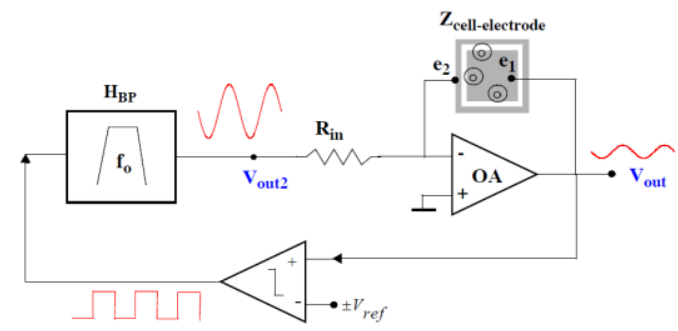

Fig. 6: Block diagram of the proposed OB implementation. Oscillations $\left(a_{o s c}\right.$, $f_{\text {osc }}$ ) will be measured at $V_{\text {out } 2}$ signal. 
oscillators. A solution to this problem consists of a non-linear element (a simple comparator) within the feedback loop in Fig. 6 to keep sustained oscillations [9-11]. This non-linear element also allows a precise control of the oscillation amplitude. On the other hand, we need to guarantee that a set of oscillation conditions can be fulfilled in order to keep oscillations alive. A simple way to implement the oscillator is including a band-pass filter in the loop, as proposed in the general circuit block in Fig. 6.

For the sake of simplicity, let us now consider the case of a second-order Band Pass Filter (BPF) and a comparator with saturation levels $\pm V_{\text {ref. }}$. This closed-loop system verifies the required properties: the system is autonomous, the nonlinearity is separable and frequency-independent, and the linear transfer function contains enough low-pass filtering to neglect the higher harmonics at the comparator output.

Choosing adequately the BPF, it can be forced that the firstorder Describing-Function (DF) equation [8] for the closedloop system of Fig. $6, N(a)+1 / H(s)=0$, has an oscillatory solution $\left(\omega_{\mathrm{osc}}, a_{o s c}\right)$, being $\omega_{\text {osc }}$ and $a_{\text {osc }}$, the oscillation frequency and amplitude, respectively. The DF function will be, in this case, $N(a)$, while $H(s)$ is the transfer function of the closed-loop system. Modelling the system mathematically, the characteristic equation can be described as:

$$
1+N(a) \cdot H(s)=0
$$

where, as previously stated, $N(a)$ is the comparator describingfunction and $H(s)$ the modified system. The general BPF transfer function will be given by,

$$
H_{B P}(s)=\frac{k_{1}^{*}\left(\omega_{o}^{*} / Q^{*}\right) s}{s^{2}+\left(\omega_{o}^{*} / Q^{*}\right) s+\omega_{o}^{* 2}}
$$

Since the corresponding SUT transfer function, $H_{z}(s)$,

$$
H_{Z}(s)=\frac{k_{2} s^{2}+k_{1}\left(\omega_{o} / Q\right) s+k_{o} \omega_{o}^{2}}{s^{2}+\left(\omega_{o} / Q\right) s+\omega_{o}^{2}}
$$

being the constant parameters $\left(\omega_{0}, Q\right.$ and $\left.k_{o}, k_{1}, k_{2}\right)$ directly related to the electrode size, technology and biological material $(f f)$. Then, the global function expression will be given by,

$$
H(s)=H_{B P}(s) \cdot H_{Z}(s)
$$

To force the oscillations, a pair of complex poles of the overall system has to be placed on the imaginary axes. The way to determine the oscillation conditions (gain, frequency and amplitude) is solving equation (1). This is equivalent to find the solution of this equation set:

$$
1+N(a) H(s)=\left(s^{2}+w_{o s c}^{2}\right)\left(s^{2}+B s+A\right)=0
$$

where the coefficients are obtained from the equation system,

$$
\begin{aligned}
& B=\left(w_{o} / Q\right)+\left(w_{o}^{*} / Q^{*}\right)+N\left(a_{o s c}\right) k_{1}^{*} k_{2}\left(w_{o}^{*} / Q^{*}\right) \\
& A+w_{o s c}^{2}=w_{o}^{2}+\left(w_{o} / Q\right)\left(w_{o}^{*} / Q^{*}\right)+w_{o}^{* 2}+N\left(a_{o s c}\right) k_{1}^{*}\left(w_{o}^{*} / Q^{*}\right) k_{1}\left(w_{o} / Q\right) \\
& B \cdot w_{o s c}=\left(w_{o}^{*} / Q^{*}\right) w_{o}^{2}+\left(w_{o}^{*} / Q^{*}\right) w_{o}^{* 2}+N\left(a_{o s c}\right) k_{1}^{*}\left(w_{o}^{*} / Q^{*}\right) k_{o} w_{o}^{2} \\
& w_{o s c}^{2} A=w_{o}^{2} w_{o}^{* 2}
\end{aligned}
$$

The output of the biological filter (the input to the nonlinear element) is approximately sinusoidal due to the band- pass characteristics of the global structure. This fact allows us to use the linear approximation stated in the describing function method [8-11] for the mathematical treatment of the non-linear element. As a result, the transfer function of this non linear element can be approximated as $N\left(a_{o s c}\right)=4 V_{\text {ref }}$ $/ \pi a_{\text {osc }}$, where $V_{\text {ref }}$ is the comparator voltage reference (whose value can be swept to obtain the adequate signal levels) and $a_{\text {osc }}$ is the amplitude of the oscillation.

For each fill-factor $f f$, there exists an oscillatory solution. As a consequence, the main oscillation parameters are function of the occupied cell-culture area. For the case described in Fig. 2, Fig 7 illustrates the frequency and amplitude of the predicted oscillations $^{1}$. Let us remark how the oscillation frequency increases monotonically in the range $[7560,7920] \mathrm{Hz}(0.16$ $\mathrm{Hz} / \mu \mathrm{m}^{2}$ of electrode area occupied by cells) and the oscillation amplitude also increases monotonically in the interval $[0,500]$ $\mathrm{mV}\left(0.2 \mathrm{mV} / \mu \mathrm{m}^{2}\right.$ of electrode area occupied by cells $)$, when the cell-electrode area overlap $\left(A_{c}\right)$ is increasing from 0 to 1 . Since the signal level in $V_{\text {out }}$ (see Fig. 6) is very small, due to electrode modelling constraints, the secondary output ( $V_{\text {out } 2}$ in Fig. 6) can be considered as the potential voltage output (whose value is related with the oscillation parameters), thus improving the dynamic range.

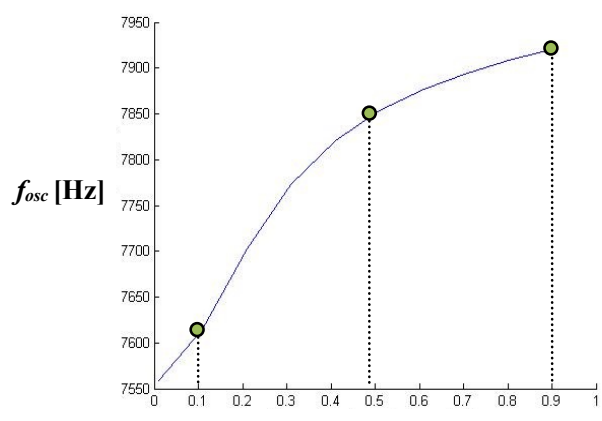

fill-factor $[\% / 100]$

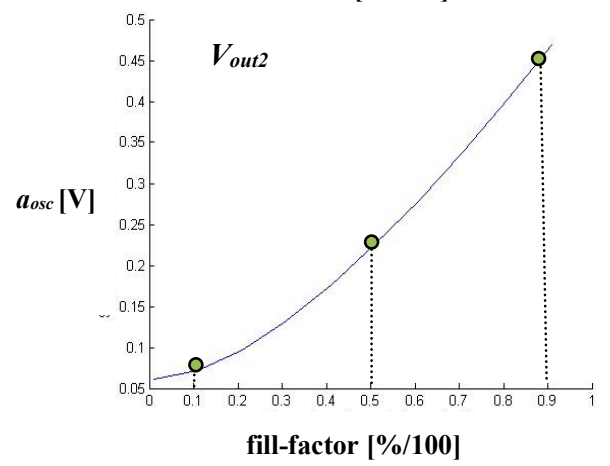

Fig. 7: Oscillation parameters obtained from cell-to-electrode area overlap defined by $f f=A / A_{c}$ ff in $[0,0,9]$. The approximated sensitivities are 0.16 $\mathrm{Hz} / \mu \mathrm{m}^{2}$ for $f_{\text {osc }}$, and $0.2 \mathrm{mV} / \mu^{2}$ for $V_{\text {out } 2}$, using a gold micro-electrode of $2500 \mu \mathrm{m}^{2}$ surface.

In this work, the above-mentioned scheme (Fig. 6) to implement the oscillator is proposed. The non-linear feedback element is connected to the "biological filter" to implement the oscillator. In this way, only the input and output of such a "biological filter" are manipulated to perform the test allowing a low intrusion in the structure.

\footnotetext{
${ }^{1}$ For every application, the sensitivity of the oscillation parameters with regard to the filter and the comparator has to be determined in order to maximize both the oscillation frequency and the dynamic range.
} 


\section{PRACTICAL CIRCUIT}

A discrete component OPAMP-based prototype was implemented at lab as a proof of concept, following the scheme proposed at Fig. $6[10,11]$. The main circuit blocks are the followings:

- A second-order active Band Pass Filter, with a cut-off frequency that can be tuned in the range of 6.6 to $13 \mathrm{kHz}$, depending on system sensitivity.

- A voltage comparator incorporating a low-pass filter, a 20 $\mathrm{dB}$ voltage amplifier and a hysteresis block for noise reduction.

- An Automated Gain Control (AGC), required for limiting the voltage amplitude applied to the electrodes. Alternatively, the AGC system can be used to easily extract useful information from the sample values.

- An analog multiplexor circuit, for channel selection, in the case of being interested in measuring multiple samples.

- A digital block based in a microcontroller for activating the bio-oscillator activity, choosing the multiplexed output, and controlling the 12-bit $\mathrm{ADC}$ for an accurate AGC operation.

A more extensive explanation about the circuit design employed can be found at [10]. In a first test approach, several loads $\mathrm{NaCl}$ saline solution concentration were set. Also, cell culture assays with different concentration were used as loads.

\section{RESULTS}

The electronic system described in paragraph IV was fully implemented and some measurements were performed at lab. Two types of experimental test using commercial electrodes from $\mathrm{AB}$ [12] were performed: first, with different $\mathrm{NaCl}$ dilutions (and no cells to sweep the conductivity) and second, testing the cell-cultures time evolution with several cell densities.

\section{A. NaCl loads}

Some solutions of $\mathrm{NaCl}$ were employed for verifying the circuit performance using saline solutions of different concentrations, corresponding to different impedances. This experiment used the $8 \mathrm{E} 1 \mathrm{~W}$ electrode (only one electrode by well) from Applied Biophysics [12], and 0,9\% $\mathrm{NaCl}$ saline solution, at dilutions of $1: 1,1: 5,1: 10,1: 20$ and 1:40. Table II shows the measured amplitudes and oscillation frequencies for the five loads. As can be seen, the oscillation frequencies are almost constant, as corresponds to a resistive behavior of the $\mathrm{NaCl}$ solutions. In Fig. 8, it can be appreciated the excellent linear agreement of the measured voltage amplitude, $a_{\text {osc }}\left(V_{\text {out } 2}\right.$ in Fig. 6), when $\mathrm{NaCl}$ conductivity decreases with the dissolution increments.

Table II: Oscillation parameters: $f_{\text {osc }}$ and $a_{o s c}$ obtained for several

\begin{tabular}{|c|c|c|}
\multicolumn{3}{|c|}{$\mathrm{NaCl}$ saline solution dilutions. } \\
\hline $\mathrm{NaCl} 0.9 \%$ & $f_{\text {osc }}[\mathrm{kHz}]$ & $a_{\text {osc }}[\mathrm{V}]$ \\
\hline $1: 1$ & 7.86 & 0.058 \\
\hline $1: 5$ & 7.93 & 0.285 \\
\hline $1: 10$ & 7.94 & 0.575 \\
\hline $1: 20$ & 7.94 & 1.050 \\
\hline $1: 40$ & 7.94 & 2.080 \\
\hline
\end{tabular}

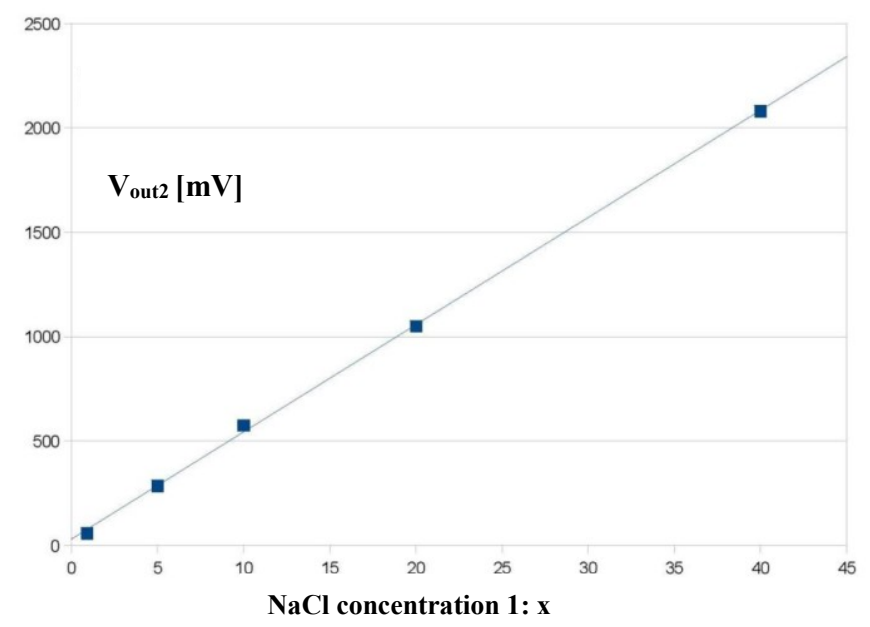

Fig. 8: Voltage amplitude at $\mathrm{V}_{\text {out2 }}$ versus $\mathrm{NaCl}$ solution concentration.

\section{B. Cell Culture loads}

Cell culture assays were also done to test the system. The eight $8 \mathrm{~W} 10 \mathrm{E}$ wells were seeded with only-medium ( 2 wells), plus 500 cells ( 2 wells), 1000 cells ( 2 wells) and 2000 cells (2 wells). Observation times were defined at 5 (P1) and 7 (P2) days. The frequency $\left(f_{\text {osc }}\right)$ and amplitude $\left(a_{o s c}\right)$ of the oscillation were measured, and summarized at table III. Fig. 9 illustrates the waveforms measured at 5 days for $V_{\text {out } 2}$ and the comparator output at the 500 cells case. $V_{\text {out } 2}$ oscillation amplitude and frequency are $1.30 \mathrm{~V}$ and $7912 \mathrm{~Hz}$, respectively. These values are increased to $1.54 \mathrm{~V}$ and $7922 \mathrm{~Hz}$ at the 2000 cells case, Fig. $10^{2}$. Finally, amplitude and oscillation frequencies at $\mathrm{P} 1$ are represented at Figures 11 and 12 respectively. It can be observed the direct linear dependence of both with the fill factor, validating our system approach.

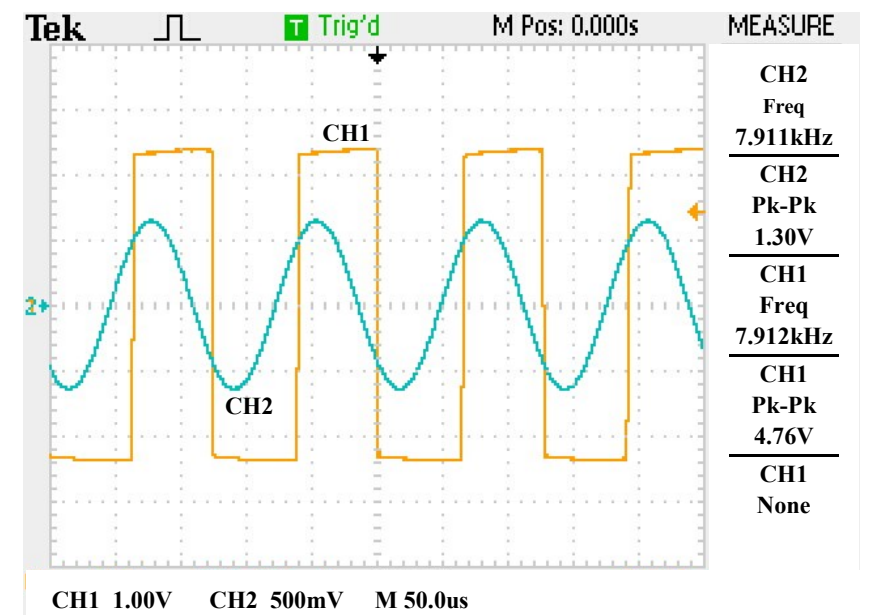

Fig. 9: Voltage waveforms of $\mathrm{V}_{\text {out2 }}(\mathrm{CH} 2)$ and comparator output $(\mathrm{CH} 1)$. 500cells case, at $\mathrm{P} 1$.

\footnotetext{
2 It can be argued that the frequency variation is too small in these experimental measurements. This is mainly due to the inclusion of an AGC, which is employed to improve the output voltage dynamic range ensuring that the oscillation amplitude in Vout (Fig. 6) is consistent with the modelling constraints. This block limits the sensibility of the frequency measurements. However, it should be noticed that just amplitude measurements are enough to validate the proposed $\mathrm{OB}$ technique.
} 


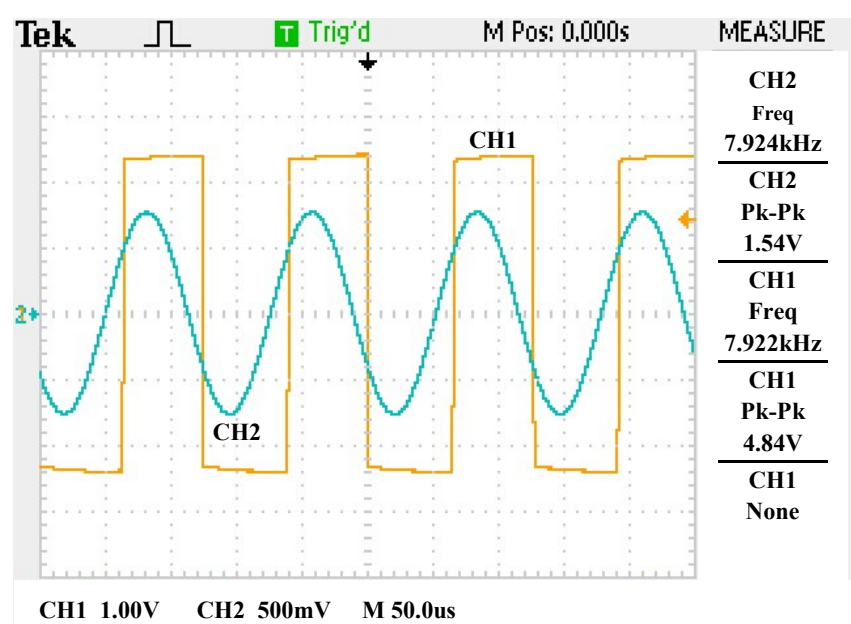

Fig. 10: Voltage waveforms of $\mathrm{V}_{\text {out2 }}(\mathrm{CH} 2)$ and comparator output $(\mathrm{CH} 1)$. 2000-cells case, at P1.

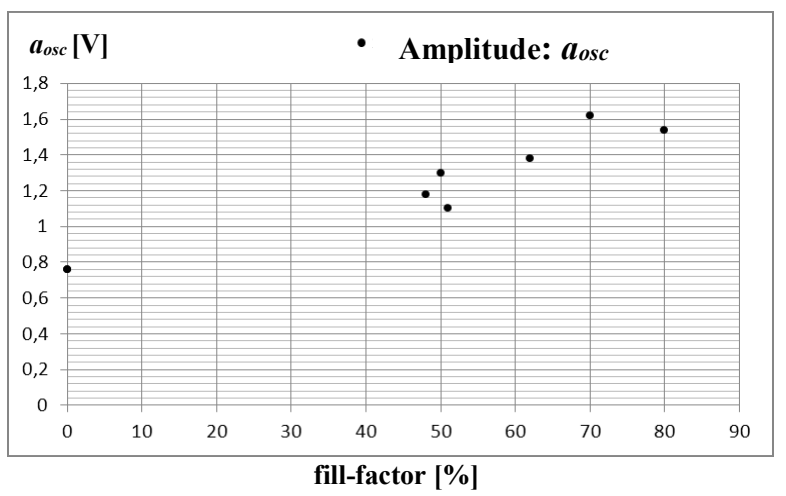

Fig. 11: Amplitude versus fill-factor (data from 5 days).

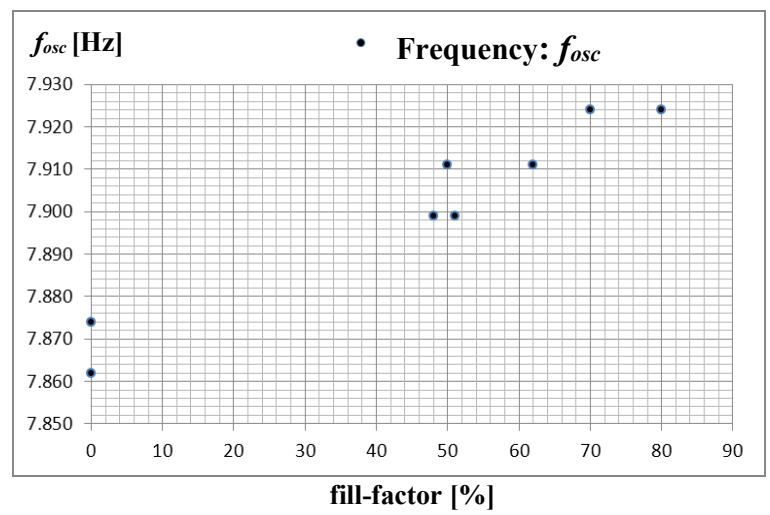

Fig. 12: Frequency versus fill-factor (data from5 days).

Table III: Electrode surface occupation $(f f)$ in \%, amplitude $\left(a_{o s c}\right)$ and frequency $\left(f_{o s c}\right)$ of oscillations obtained from test with living cells.

\begin{tabular}{|l|c|c|c|c|c|c|}
\hline & \multicolumn{3}{|c|}{$P 1: 5$ days-grow } & \multicolumn{3}{c|}{$P 2: 7$ days-grow } \\
\hline Well $-\mathrm{n}$-cells & $\mathrm{ff}(\%)$ & $a_{\text {osc }}[\mathrm{V}]$ & $f_{\text {osc }}[\mathrm{kHz}]$ & $\mathrm{ff}(\%)$ & $a_{\text {osc }}[\mathrm{V}]$ & fosc $\left._{\text {ofHz }}\right]$ \\
\hline $1-$ Med & 0 & 0.76 & 7.862 & 0 & 0.76 & 7.860 \\
\hline $2-500$ & 58 & 1.30 & 7.911 & 79.5 & 1.42 & 7.918 \\
\hline $3-1000$ & 72 & 1.62 & 7.924 & 90.5 & 1.66 & 7.937 \\
\hline $4-2000$ & 51 & 1.10 & 7.899 & Death c. & - & - \\
\hline $5-$ Med & 0 & 0.76 & 7.874 & 0 & 0.74 & 7.860 \\
\hline $6-500$ & 62 & 1.38 & 7.911 & 95 & 1.54 & 7.930 \\
\hline $7-1000$ & 48 & 1.18 & 7.899 & 56 & 1.22 & 7.911 \\
\hline $8-2000$ & 80 & 1.54 & 7.924 & 100 & 1.66 & 7.937 \\
\hline
\end{tabular}

\section{CONCLUSIONS}

This paper presents a system for cell-culture monitoring using the oscillation based concept. A simple topology based on a non-linear element embedded in a feedback loop is employed to convert the Cell-Culture Under Test (CCUT) into a suitable bio-oscillator. The technique does not need a specific excitation signal as the standard ECIS technique requires. Furthermore, a key feature of this proposed method is that the results of the measurements are robust enough to avoid the need to carry out a statistical evaluation of multiple samples as it is usual in most ECIS techniques. A practical implementation with commercial circuit components has been proposed to prove the concept, from which simulation and experimental results validate the predictions. Test performance with cell cultures has been demonstrated, obtaining calibration curves for fill factor that allows a non-invasive continuous real-time monitoring of cell culture assays at low cost. The procedure used herein can be generalized and adapted to any impedance measurement problem.

\section{ACKNOWLEDGMENT}

This work was supported in part by the Spanish founded Projects: TEC2011-28302 and TEC2013-46242-C3-1-P: Integrated Microsystem for Cell-Culture Assays. The authors also thanks to research team of Dr. Pablo Huertas, from Andalusian Molecular Biology and Regenerative Medicine Centre (Cabimer), Sevilla, Spain, for the help with cell culture assays.

\section{References}

[1] S. Grimnes, S. and O. Martinsen, "Bio-impedance and Bioelectricity Basics," Second edition. Academic Press, Elsevier. 2008.

[2] H. Wissenwasser, M. J. Vellekoop and R. Heer, "Signal Generator for Wireless Impedance Monitoring of Microbiological Systems," IEEE, TIM-60, nº 6, pp: 2039-2046. 2011.

[3] D. Borkholder, Cell-Based Biosensors Using Microelectrodes. PhD Thesis, Stanford University. 1998.

[4] A. Manickam et al,"A CMOS Electrochemical Impedance Spectroscopy (EIS) Biosensor Array," IEEE BioCAS, nº 6, pp:379-390. 2010.

[5] I. Giaever and Ch. Keese: "Use of Electric Fields to Monitor the Dynamical Aspect of Cell Behaviour in Tissue Culture," IEEE BME-33, n 2 . pp: 242-247. 1986.

[6] P. Daza, A. Olmo, D. Cañete and A. Yúfera, "Monitoring Living Cell Assays with Bio-Impedance Sensors," Sensors and Actuators B, 176, pp: 605-610. 2013.

[7] X. Huang et al., "Simulation of Microelectrode Impedance Changes Due to Cell Growth," IEEE Sensors Journal, vol.4, n 5. pp: 576-583. 2004

[8] G. Huertas, D. Vázquez, A. Rueda and J. L. Huertas, Oscillation-Based Test in Mixed-Signal Circuits. Springer. 2006.

[9] P. Fleischer et al, "A Switched Capacitor Oscillator with Precision Amplitude Control and Guaranteed Start-Up” IEEE JSSC-20, no2. 1985.

[10] A. Maldonado, J. Olmo, G. Huertas and A. Yúfera. A Cell-Culture RealTime Monitoring System. Texas Instruments. First place at "Analog Design Contest in Europe" (www.ti.com/tiic-eu). 2013.

[11] G. Huertas, A. Maldonado, A. Yúfera, A. Rueda and J. L. Huertas, "Oscillation-Based Test Applied to Cell Culture Monitoring," IEEE Sensors 2013, pp: 842-845. Baltimore, USA. 2013.

[12] J. Müller, C. Thirion, M. W. Pfafflal, "Electric cell-substrate impedance sensing (ECIS) based real-time measurement of titer dependent cytotoxicity induced by adenoviral vectors in an IPI-2I cell culture model," Biosensors and Bioelectronics 26, pp: 2000-2005. 2011. 